\title{
Results of Endovascular Therapy of Ureteroarterial Fistulas: A Single Center Experience
}

Bjoern Simon ( $\nabla$ bjoern.simon@uniklinik-freiburg.de)

Department of Radiology, Medical Center - University of Freiburg, Faculty of Medicine, University of Freiburg, Germany https://orcid.org/0000-0003-0418-9149

\section{Jakob Neubauer}

Department of Radiology, Medical Center - University of Freiburg, Faculty of Medicine, University of Freiburg, Germany

\section{Martin Schoenthaler}

Department of Urology, Medical Center - University of Freiburg, Faculty of Medicine, University of Freiburg, Germany

\section{Simon Hein}

Department of Urology, Medical Center - University of Freiburg, Faculty of Medicine, University of Freiburg, Germany

\section{Fabian Bamberg}

Department of Radiology, Medical Center - University of Freiburg, Faculty of Medicine, University of Freiburg, Germany

\section{Lars Maruschke}

Diagnostic Radiology, Pediatric Radiology and Interventional Radiology, St.-Josefs-Krankenhaus, Freiburg

\section{Research Article}

Keywords: Ureteroarterial fistula, arterioureteral fistula, endovascular therapy, hematuria, ureteral catheterization, stent graft, coil embolization

Posted Date: March 6th, 2021

DOl: https://doi.org/10.21203/rs.3.rs-266941/v1

License: (c) (i) This work is licensed under a Creative Commons Attribution 4.0 International License. Read Full License

Version of Record: A version of this preprint was published at CVIR Endovascular on April 17th, 2021. See the published version at https://doi.org/10.1186/s42155-021-00226-6. 


\section{Abstract}

\section{Background}

Ureteroarterial fistula (UAF) is a rare but potentially life threatening disease. The aim of this study was to evaluate the outcome of endovascular therapy for UAF treatment.

\section{Methods}

This retrospective case series evaluates a single center experience of percutaneous stent graft angioplasty and/or coil embolization for UAF. Patient follow-up included technical and early clinical success, complications and revisional procedures.

\section{Results}

We identified 17 UAF in 16 consecutive patients (12 male, 4 female, mean age $69.8 \pm 11.3$ years) who underwent endovascular UAF therapy at our tertiary hospital. All patients presented with hematuria. 5/17 presented with flank pain, in 7 of 17 cases patients were in hypovolemic shock. Risk factors of UAF included chronic indwelling ureteral stents in all fistulas, pelvic exstirpatory surgery in 13 cases. In 6 cases stent grafts were placed from the common iliac artery (CIA) to the external iliac artery (EIA) following coil embolization of the proximal internal iliac artery (IIA). Stent graft placement without previous coil embolization was performed in 10 fistulas. In one case only coil embolization of the IIA was performed. Mean follow-up was 654 (range: 1 - 3269) days. All procedures were technically successful and no procedure related deaths occurred during follow-up. During the initial hospital stay hematuria disappeared in 14/17 cases. Overall, four patients suffered recurrent hematuria, which in three cases resolved after a secondary intervention. One recurrent UAF related death occurred during follow-up 229 days after initial treatment.

\section{Conclusion}

Endovascular therapy proves to be a safe and efficient method in UAF treatment.

\section{Evidence-based medicine}

Level 4, case series.

\section{Background}

Ureteroarterial fistula (UAF) is a rare but potentially life threatening clinical entity first described in 1908 by Moschcowitz (Moschcowitz, 1908). UAF is classified into primary (15\%) and secondary ( $85 \%$ ) lesions (Pillai et al., 2015). Primary fistulas are mainly seen in combination with aortoiliac aneurysmal disease. Secondary fistulas typically occur after pelvic surgery for malignancy with or without radiation therapy, or after vascular surgery with synthetic grafting (Bergqvist et al., 2001; Luther et al., 2014). The most 
common risk factor to develop an UAF is the presence of a chronic indwelling ureteral stent (Das et al., 2016). This is possibly due to the fact that mechanical fixation of the ureter triggers inflammation and fibrosis in the adjacent pulsating artery (Horie et al., 2019).

The most common location of UAF is the crossing of the distal left common iliac artery (CIA) and the ureter (Das et al., 2016; van den Bergh et al., 2009). The leading symptom is gross hematuria (Bergqvist et al., 2001; Das et al., 2016; Krambeck et al., 2005). UAF is a rare condition; however, since the increase in life expectancy in patients suffering of pelvic malignancies, this condition is observed more often (Das et al., 2016; Fox et al., 2011).

Different treatment options including surgery or surgery combined with transarterial embolization have been described (Fox et al., 2011; Krambeck et al., 2005; van den Bergh et al., 2009). More recently, stent graft placement has been reported as an effective alternative treatment; however, only small series using a variety of different, mostly self-expanding stent grafts or iliac extensions from aortic bifurcation grafts have been reported (Fox et al., 2011; Guntau et al., 2017; Krambeck et al., 2005; Okada et al., 2013).

Thus, the purpose of the study was to evaluate the safety and effectiveness of endovascular therapy in UAF and to establish an interventional radiological therapy regime for these rare but critical cases.

\section{Methods}

IRB approval was obtained on June 25, 2020 by the institutional review board of the University of Freiburg (\#368/20).

Informed consent was waived due to our analysis' retrospective nature.

We included all consecutive patients who were referred for endovascular therapy for UAF at our institution from November 2005 to March 2020. Patient characteristics, comorbidities, and clinical presentation were reviewed from medical records. The clinical diagnosis of UAF and compromised side determination was based on pulsatile ureteral bleeding on cystoscopy and/or in retrograde pyelogram by the referring urologist.

Radiological diagnostic work up included either contrast-enhanced computed tomography (CECT), unenhanced computed tomography (UECT) or conventional angiography. Fistula location was identified from cystoscopy by the site of ostial hemorrhage. CT was useful to rule out renal hemorrhages and for planning of the endovascular treatment. Thereafter, patients underwent conventional catheter angiography. All angiographic procedures were performed under local anesthesia via a percutaneous transfemoral approach. First a 5 French $(\mathrm{Fr})$ angiographic sheath was placed using Seldinger's technique. Angiograms were obtained with manual contrast injection via a $5 \mathrm{Fr}$ angiographic catheter.

For stent graft placement, the $5 \mathrm{Fr}$ sheath was replaced by a larger sheath $(7 \mathrm{Fr}-14 \mathrm{Fr})$ and stiff wire was placed. Usually, the UAF was treated with percutaneous covered stent graft placement into the iliac axis covering the site of the fistula. In a minority of cases fistulas had additional coil embolization of the 
internal iliac artery (IIA). Stent size was chosen to match the individual patient's anatomy. In one case the bleeding site was localized solely in the IIA therefore the fistula was treated by coil embolization of the IAA alone. The procedure was considered complete and technically successful after a final angiogram confirmed the correct stent placement at the target location and/or the sufficient embolization of the IIA. Early clinical success was defined as freedom of hematuria in the current hospital stay. A descriptive analysis was performed. Continuous data were summarised by arithmetic mean, and range. Categorical data were summarised by the total number of patients in each category. Relative frequencies are displayed as valid percentages.

\section{Results}

\section{Clinical findings}

In the study period, 17 UAFs with a total of 16 consecutive predominantly male patients were included in the analysis ( $75 \%$ male, mean age $69.8 \pm 11.3$ years). All patients presented with hematuria. Approximately one third of patients (31.3\%) exhibited additional flank pain, in seven cases $(41.2 \%)$ patients were in hypovolemic shock. In $94.1 \%$ of the cases patients presented with acute urinary retention. Half of the patients had chronic nephropathy. Risk factors of UAF included chronic indwelling ureteral stents in all fistulas, pelvic exstirpatory surgery in 13 cases (76.5\%) and history of pelvic or genitourinary malignancies including colorectal cancer $(n=10)$, uterine/cervical cancer $(n=3)$, bladder cancer $(n=1)$, testicular cancer $(n=1)$ and prostate cancer $(n=1)$. One patient had radiotherapy as primary treatment, one patient was under active surveillance and the only patient who did not suffer of a malignancy but of Ormond's disease had cortisone therapy as primary treatment. Fourteen of 15 patients with cancer had adjuvant radiation therapy and chemotherapy. Five patients (31.3\%) suffered of vasculopathies, two had severe claudication (Rutherford III), one had a right critical limb ischemia (Rutherford $\mathrm{V}$ ), one had abdominal aortic aneurysmal disease and one patient had documented peripheral arterial occlusive disease with aortofemoral bypass. Patient characteristics are summarized in Table 1.

\section{Imaging}

Urologists were able to confirm signs of ureteral bleeding and correctly located the site of bleeding by using cystoscopy in all patients. In 10 cases the first radiological diagnosis was done by CECT, in 4 cases direct catheter angiography in readiness to intervene followed urological diagnosis confirmation, and in 3 cases an unenhanced CT was performed prior to angiography. CECT directly identified the fistula only in two cases (of 10 cases who received preinterventional CECT) with extravasation into the ureter. Angiography was positive in 3 of 17 cases. CT or angiography identified the crossing point between ureter and iliac artery in all patients. In 10 cases, the UAF was located at the CIA, in 3 cases each the IIA or the external iliac artery (EIA) was compromised respectively; in one exceptional case the fistula was located at the aortofemoral bypass graft.

\section{Procedures}


We inserted stent grafts without previous coil embolization in 10 fistulas. In 6 cases we performed coil embolization of the proximal IIA prior to stent graft implantation. In one case the UAF was located solely in the IIA and was therefore treated by coil embolization alone. In three cases a secondary procedure was necessary due to recurrent bleeding during the hospital stay: In two cases stent graft extension and additional coil embolization of the IIA was needed and in one fistula IAA coiling alone was sufficient. Unrelated to the UAF but because of ongoing aneurysm growth due to aneurysmosis of the aortoiliac territory a secondary stent graft extension into the proximal CIA was required in one case.

Different types of balloon-expandable and self-expandable stent grafts were used: Advanta V12 (Atrium Medical, NH, USA) in 7 cases, Fluency (Bard Peripheral Vascular, Tempe, AZ, USA) in 5, Gore Excluder endograft (Gore, Flagstaff, AZ, USA) in 3, Viabahn endograft (W. L. Gore, Flagstaff, AZ, USA) in 3 and Endurant II endograft (Medtronic Vascular, Santa Rosa, CA, USA) in 1. In 16 of 17 cases puncture site was closed by a closure device: In 4 cases FemoSeal ${ }^{T M}$ (St. Jude Medical, Plymouth, MN, USA) was used, in 9 cases Perclose ProGlide (Abbott Vascular, Abbott Park, IL, USA) was applied, StarClose (Abbott Vascular, Abbott Park, IL, USA) was used twice and in one case Prostar XL (Abbott Vascular, Santa Clara, CA, USA) was employed.

All but two patients were put on acetylic salicylic acid $100 \mathrm{mg} /$ day immediately following UAF treatment, 6 patients received additional 12 weeks of clopidogrel $75 \mathrm{mg} /$ day and 1 patient for 6 weeks. One patient had 12 weeks of clopidogrel $75 \mathrm{mg} /$ day combined with previously started oral anticoagulation. The patient who had coil embolization only did not receive any antiplatelet therapy. Diagnostic and technical data are shown in Table 2.

\section{Outcomes}

Mean follow-up was 654 (range: 1-3269) days and four patients (23.5\%) were lost to follow-up between day one and 11 days. Among the remaining cases, no primary procedure-related deaths occurred during follow-up. Similarly, 30-day mortality rate was zero. All procedures were technically successful. During current hospital stay hematuria disappeared in $82.4 \%$ of the cases $(n=14)$. After a more extensive re-do intervention hematuria resolved in the remaining cases.

Recurrent hematuria happened in four cases, three of which occurred during the current hospital stay (8, 8 and 13 days after initial treatment). Two could successfully be treated via secondary stent extension over the iliac crossing plus additional coil embolization of the IIA (case \#7 and \#11). In case \#16 where the fistula was located at the ureteral crossing of the aortofemoral bypass graft secondary coil embolization of the IIA alone was sufficient. There was one recurrent UAF related death 229 days after initial treatment (case \#3). In this patient, a re-opening of the $\square$ stula was found, caused by attempted interventional thrombectomy for critical limb ischemia. Periprocedural complications happened in two cases - in case \#3 the stent graft could not fully be unfolded and in case \#12 the Prostar XL system did not work after placement of a $14 \mathrm{Fr}$-sheath which led to prolonged manual compression. Stent thrombosis happened in 3 cases (1, 2 and 58 days after initial treatment). Two occlusions could have been managed via interventional thrombectomy with or without thrombolysis (case \#3 and case \#14). In case \#12 where the 
failed closure device led to immediate thrombosis the day after the patient was transferred to surgical thrombectomy. Septicemia was found in 5 of 17 cases (29.4\%) at the point of intervention, in follow up there was no history of stent graft infections detected in those patients. During follow-up, one patient died of the underlying disease ( 576 days after the initial treatment). Early and long-term outcomes are summarized in Table 3.

\section{Discussion}

To our knowledge we conduct the largest retrospective interventional radiological cohort study of cases with secondary UAFs from a single center.

The main symptom in our UAF patients was hematuria in all cases ranging from non-life-threatening transient hemorrhage to hypovolemic shock (in $41.2 \%$ of the cases). The need for blood transfusions and/or inotropic therapy is common. Flank pain and fever are further occurring as well (Heers et al., 2018).

Our diagnostic work up showed that in the minority of cases an active bleeding site could be located via CECT ( $2 / 10$ cases; $20.0 \%$ ) or angiography ( $3 / 17$ cases; $17.7 \%)$. This confirms the findings of Guntau et al. with two detected UAFs in eight patients by CECT alone (Guntau et al., 2017). However, a negative CECT or angiogram does not rule out the diagnosis, therefore cystoscopy is used for confirmation of UAF and detecting the site of the fistula (Krambeck et al., 2005). Nevertheless, CECT proves to be crucial for planning the interventional approach, to locate the ureteral crossing and to rule out any other bleeding sites. Provocative angiography as an invasive imaging tool has been described as an effective procedure to demonstrate the active hemorrhage (Das et al., 2016; Fox et al., 2011; Guntau et al., 2017; van den Bergh et al., 2009). However, the risk of triggering extensive bleeding or to re-open a currently clotted fistula seems unnecessarily high. Positive CECT and angiography is shown in Fig. 1.

In the therapeutic management, there has been a paradigm shift from a surgical approach, which was still the standard of care in 2004, toward interventional angiography (Bergqvist et al., 2001; Fox et al., 2011; Madoff et al., 2004). Patients typically carry a high risk for complications due to previous extensive surgery and radiation therapy which lead to adhesions, bbrosis, and frail tissue (Heers et al., 2018). In patients with UAF to the IIA only, arterial coil embolization can be considered. Most of the UAF involve EIA or CIA, so covered stent treatment is necessary (Muraoka et al., 2006; van den Bergh et al., 2009). Stent graft treatment is less invasive and offers rapid bleeding control (Patel et al., 2014).

Endovascular therapy offers high technical and early clinical success rates $(100 \%$ and $82.4 \%$ in our cohort). Severe complications like stent occlusions or stent graft infections are rare (Malgor et al., 2012; Okada et al., 2013). Stent thrombosis is one important postinterventional complication: In our experience major risk factors for developing stent thrombosis were underlying vasculopathy or advanced tumor disease with extrinsic stenosis of iliac vessels. Too maintain the risk for stent occlusions as low as possible patients should be put on single anti platelet therapy after the procedure. Periprocedural complications can be decreased by using equipment with high personal experience level. When using 
large sheaths it is often necessary to use a closure device. In one case device failure led to prolonged manual compression and stent occlusion the day after. Literature already disclosed controversies after use of Prostar XL (Maniotis et al., 2017; Power et al., 2019).

In spite of a high number of reported urinary tract infections which sometimes lead to septicemia in these patients, the incidence of secondary stent graft infections seems to be overrated (Darcy, 2009). Stent graft placement in an infectious site is critical. Nevertheless, UAF typically requires urgent treatment. It is therefore recommended that these patients must receive perioperative antibiotic treatment (Hong et al., 2016). In the current study none of the patients suffered from morbidity due to stent graft infections during their postinterventional course. In the so far largest published multi-center study by Heers et al. including 24 patients only one patient developed stent infection. This matches previously published experience (Malgor et al., 2012).

In the current series, recurrent bleedings developed after insufficient coverage of the fistula during the initial intervention and were manageable by a secondary treatment. None of these patients received secondary surgical treatment for recurrent hemorrhage. Additional risk factors included complex underlying situations with severe vasculopathy or stenosing tumor burden. Following our experience, we suggest to cover the ureteral crossing from the CIA to the EIA by a stent graft with prior coil embolization of the IIA. IIA coil embolization prevents retrograde perfusion of the UAF through the gluteal arteries, but also lowers the risk of recurrent bleedings - eg., in cases of fistulas from the proximal IIA. Embolization of the IIA alone would not be sufficient for UAF treatment (Massmann et al., 2020). It is crucial to detect fistula recurrence. In our series recurrent hematuria happened in $4 / 17$ cases $(23.5 \%)$, one of them died due to recurrence of the UAF (5.9\%). Three recurrent fistulas could successfully be treated in a revisional endovascular procedure. Guntau et al. identified recurrent hematuria in one of eight patients. This patient could also successfully be treated with a secondary endovascular treatment, a combination of mild dilatation of the native iliac artery and a short distal landing zone seemed to be the cause (Guntau et al., 2017). Van den Bergh et al. found in their review of various procedures for the treatment of UAF a recurrent fistula related mortality of 18 out of 139 patients (13\%) (van den Bergh et al., 2009). In our cohort one patient died consecutively due to recurrent hemorrhage 229 days after initial treatment. This patient had a large pelvic tumor mass which led to external compression and consecutive stenosis of the iliac vessels. The inserted stent graft unfolded incompletely during the initial procedure and stent thrombosis occurred during the postinterventional course. This led to a secondary procedure to treat acute lower limb ischemia, which resulted in reopening of the UAF with consecutive bleeding (Fig. 2).

Our study is limited by its retrospective nature and despite being the largest single center endovascular experience so far to our knowledge, by the small number of cases. In addition, follow-up periods varied substantially.

\section{Conclusions}


In view of the low incidence of this disease and continuously evolving diagnostic and therapeutic tools, it seems difficult to establish evidence-based recommendations for these patients. Nevertheless, both urologists and interventional radiologists seem to be well aware of the special features of this rare disease (Heers et al., 2018).

In conclusion, endovascular therapy for UAF is a safe and effective treatment option to prevent acute hemorrhage-related death. Close and long follow-up is crucial to timely perform additional treatments if necessary (Okada et al., 2013).

\section{Abbreviations}

CECT

Contrast-enhanced computed tomography

CIA

Common iliac artery

EIA

External iliac artery

$\mathrm{Fr}$

French

IIA

Internal iliac artery

UAF

Ureteroarterial fistula

UECT

Unenhanced computed tomography

\section{Declarations}

\section{Ethical approval and consent to participate}

All procedures performed in studies involving human participants were in accordance with the ethical standards by the institutional review board of the University of Freiburg (\#368/20). and with the 1964 Helsinki declaration and its later amendments. Informed consent was waived due to our analysis' retrospective nature.

\section{Consent for publication}

Not applicable.

\section{Availability of data and materials}

All data generated or analysed during this study are included in this published article. 


\section{Competing interests}

BS is holding stocks of Medtronic plc. The other authors declare that they have no conflict of interest.

\section{Funding}

This study was not supported by any funding.

\section{Author's contributions}

BS: Conceptualization, Methodology, Data Curation, Writing. JN: Review \& Editing. MS: Supervision, Review \& Editing. SH: Review \& Editing. FB: Supervision, Review \& Editing. LM: Conceptualization \& Supervision. The authors read and approved the final manuscript.

\section{Acknowledgements}

Not applicable.

\section{References}

1. Bergqvist, D., Pärsson, H., Sherif, A., 2001. Arterio-ureteral Fistula - a Systematic Review. European Journal of Vascular and Endovascular Surgery 22, 191-196. https://doi.org/10.1053/ejvs.2001.1432

2. Darcy, M., 2009. Uretro-Arterial Fistulas. Techniques in Vascular and Interventional Radiology 12, 216-221. https://doi.org/10.1053/j.tvir.2009.09.005

3. Das, A., Lewandoski, P., Laganosky, D., Walton, J., Shenot, P., 2016. Ureteroarterial fistula: A review of the literature. Vascular 24, 203-207. https://doi.org/10.1177/1708538115585261

4. Fox, J.A., Krambeck, A., McPhail, E.F., Lightner, D., 2011. Ureteroarterial Fistula Treatment With Open Surgery Versus Endovascular Management: Long-Term Outcomes. Journal of Urology 185, 945-950. https://doi.org/10.1016/j.juro.2010.10.062

5. Guntau, M., Hegele, A., Rheinheimer, S., Hofmann, R., Mahnken, A.H., 2017. Balloon-Expandable Stent Graft for Treating Uretero-lliac Artery Fistula. Cardiovasc Intervent Radiol 40, 831-835. https://doi.org/10.1007/s00270-017-1586-4

6. Heers, H., Netsch, C., Wilhelm, K., Secker, A., Kurtz, F., Spachmann, P., Viniol, S., Hofmann, R., Hegele, A., 2018. Diagnosis, Treatment, and Outcome of Arterioureteral Fistula: The Urologist's Perspective. Journal of Endourology 32, 245-251. https://doi.org/10.1089/end.2017.0819

7. Hong, S.-Y., Noh, M., Ko, G.-Y., Han, Y., Kwon, H., Kwon, T.-W., Cho, Y.-P., 2016. Management Strategy for Ureteral-Iliac Artery Fistula. Annals of Vascular Surgery 36, 22-27. https://doi.org/10.1016/j.avsg.2016.02.033

8. Horie, K., Fujiwara, T., Satoyoshi, K., Munehisa, M., Inoue, N., 2019. Endovascular treatment of ureteroarterial fistula using a covered stent, evaluated by intravascular ultrasound: a case report. 
CVIR Endovasc 2, 16. https://doi.org/10.1186/s42155-019-0060-6

9. Krambeck, A.E., DiMarco, D.S., Gettman, M.T., Segura, J.W., 2005. Ureteroiliac artery fistula: Diagnosis and treatment algorithm. Urology 66, 990-994. https://doi.org/10.1016/j.urology.2005.05.036

10. Luther, B., von Lilien-Waldau, V., Mamopoulos, A., Katoh, M., Friedrich, M., Weinknecht, S., Lent, V., 2014. Die uretero-arterielle Fistel - Ursachen, Diagnostik und Therapieergebnisse. Aktuel Urol 45, 204-208. https://doi.org/10.1055/s-0034-1376953

11. Madoff, D.C., Gupta, S., Toombs, B.D., Skolkin, M.D., Charnsangavej, C., Morello, F.A., Ahrar, K., Hicks, M.E., 2004. Arterioureteral fistulas: a clinical, diagnostic, and therapeutic dilemma. AJR Am J Roentgenol 182, 1241-1250. https://doi.org/10.2214/ajr.182.5.1821241

12. Malgor, R.D., Oderich, G.S., Andrews, J.C., McKusick, M., Kalra, M., Misra, S., Gloviczki, P., Bower, T.C., 2012. Evolution from open surgical to endovascular treatment of ureteral-iliac artery fistula. Journal of Vascular Surgery 55, 1072-1080. https://doi.org/10.1016/j.jvs.2011.11.043

13. Maniotis, C., Andreou, C., Karalis, I., Koutouzi, G., Agelaki, M., Koutouzis, M., 2017. A systematic review on the safety of Prostar XL versus ProGlide after TAVR and EVAR. Cardiovasc Revasc Med 18, 145-150. https://doi.org/10.1016/j.carrev.2016.11.004

14. Massmann, A., Fries, P., Shayesteh-Kheslat, R., Buecker, A., Stöckle, M., Niklas, C., 2020. Lifethreatening arterioureteral fistula treatment by endovascular complete anatomic iliac artery bifurcation reconstruction. Journal of Vascular Surgery Cases and Innovative Techniques 6, 199204. https://doi.org/10.1016/j.jvscit.2020.01.012

15. Moschcowitz, A.V., 1908. SIMULTANEOUS LIGATION OF BOTH EXTERNAL ILIAC ARTERIES FOR SECONDARY HEMORRHAGE FOLLOWING BILATERAL URETEROLITHOTOMY: Annals of Surgery 48, 872-875. https://doi.org/10.1097/00000658-190812000-00009

16. Muraoka, N., Sakai, T., Kimura, H., Kosaka, N., Itoh, H., Tanase, K., Yokoyama, O., 2006. Endovascular Treatment for an Iliac Artery-Ureteral Fistula with a Covered Stent. Journal of Vascular and Interventional Radiology 17, 1681-1685. https://doi.org/10.1097/01.RVI.0000236713.46897.05

17. Okada, T., Yamaguchi, M., Muradi, A., Nomura, Y., Uotani, K., Idoguchi, K., Miyamoto, N., Kawasaki, R., Taniguchi, T., Okita, Y., Sugimoto, K., 2013. Long-term Results of Endovascular Stent Graft Placement of Ureteroarterial Fistula. Cardiovasc Intervent Radiol 36, 950-956. https://doi.org/10.1007/s00270012-0534-6

18. Patel, D., Kumar, A., Ranganath, P., Contractor, S., 2014. Endovascular treatment of arterio-ureteral fistulae with covered stents: Case series and review of the literature. SAGE Open Medical Case Reports 2, 2050313X1454809. https://doi.org/10.1177/2050313X14548094

19. Pillai, A.K., Anderson, M.E., Reddick, M.A., Sutphin, P.D., Kalva, S.P., 2015. Ureteroarterial Fistula: Diagnosis and Management. American Journal of Roentgenology 204, W592-W598. https://doi.org/10.2214/AJR.14.13405

20. Power, D., Schäfer, U., Guedeney, P., Claessen, B.E., Sartori, S., Sorrentino, S., Lefèvre, T., Kupatt, C., Tchetche, D., Dumonteil, N., Webb, J.G., Colombo, A., Windecker, S., Berg, J.M., Hildick-Smith, D., Boekstegers, P., Linke, A., Tron, C., Van Belle, E., Asgar, A.W., Jeger, R., Sardella, G., Hink, U., Husser, O., 
Grube, E., Lechthaler, I., Wijngaard, P., Anthopoulos, P., Deliargyris, E.N., Bernstein, D., Hengstenberg, C., Mehran, R., Dangas, G.D., 2019. Impact of percutaneous closure device type on vascular and bleeding complications after TAVR: A post hoc analysis from the BRAVO-3 randomized trial. Catheter Cardiovasc Interv ccd.28295. https://doi.org/10.1002/ccd.28295

21. van den Bergh, R.C.N., Moll, F.L., de Vries, J.-P.P.M., Lock, T.M.T.W., 2009. Arterioureteral Fistulas:

Unusual Suspects-Systematic Review of 139 Cases. Urology 74, 251-255.

https://doi.org/10.1016/j.urology.2008.12.011

\section{Tables}

Due to technical limitations, table 1-3 is only available as a download in the Supplemental Files section.

\section{Figures}




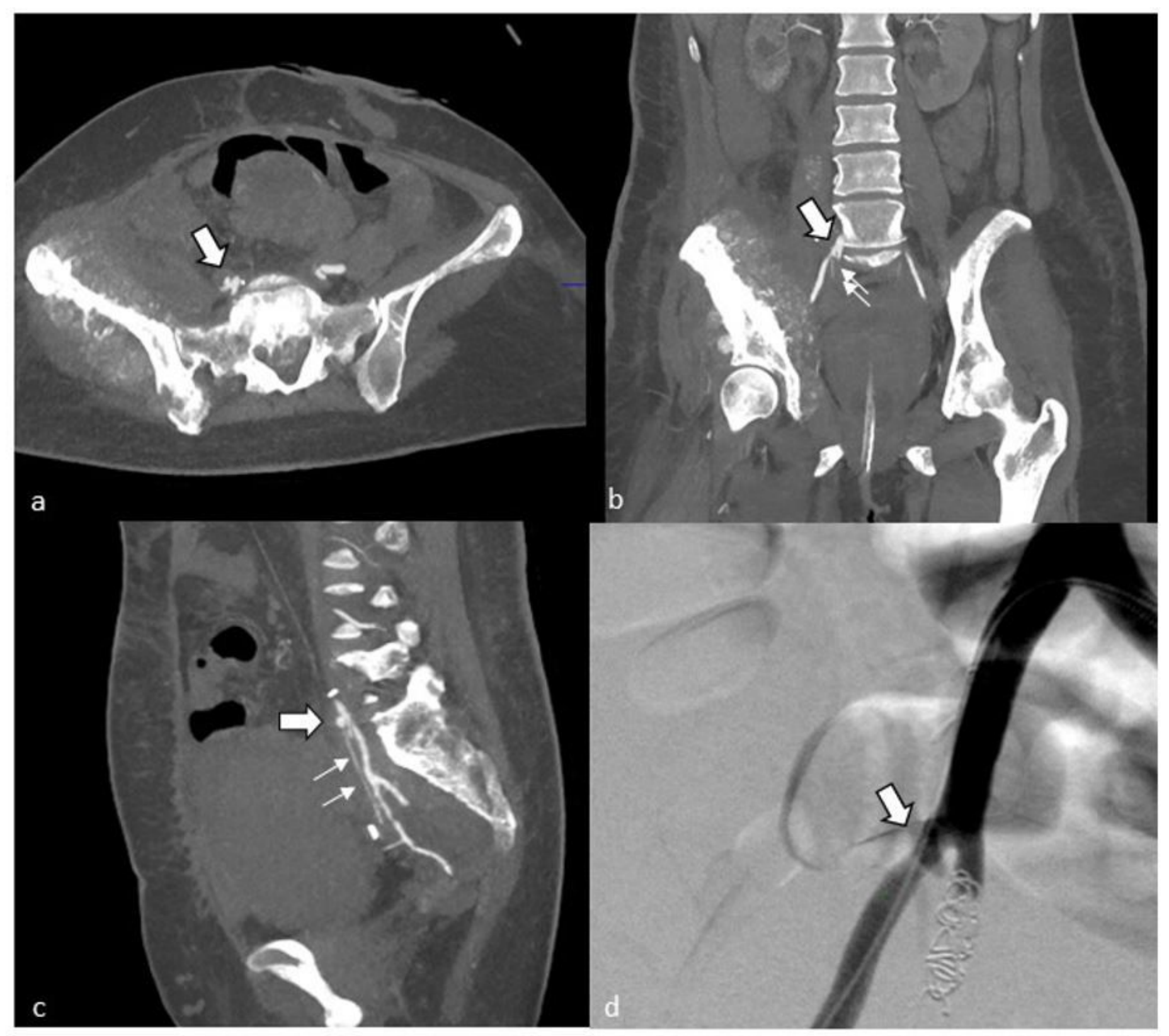

\section{Figure 1}

Case \#1: Contrast enhanced computed tomography confirmed the ureteroarterial fistula (large arrows) and demonstrated even extravasation into the right ureter (small arrows) $(a, b, c)$. Angiography shows a defect at the iliac junction (large arrow). Coil embolization of the internal iliac artery has already been performed (d). 


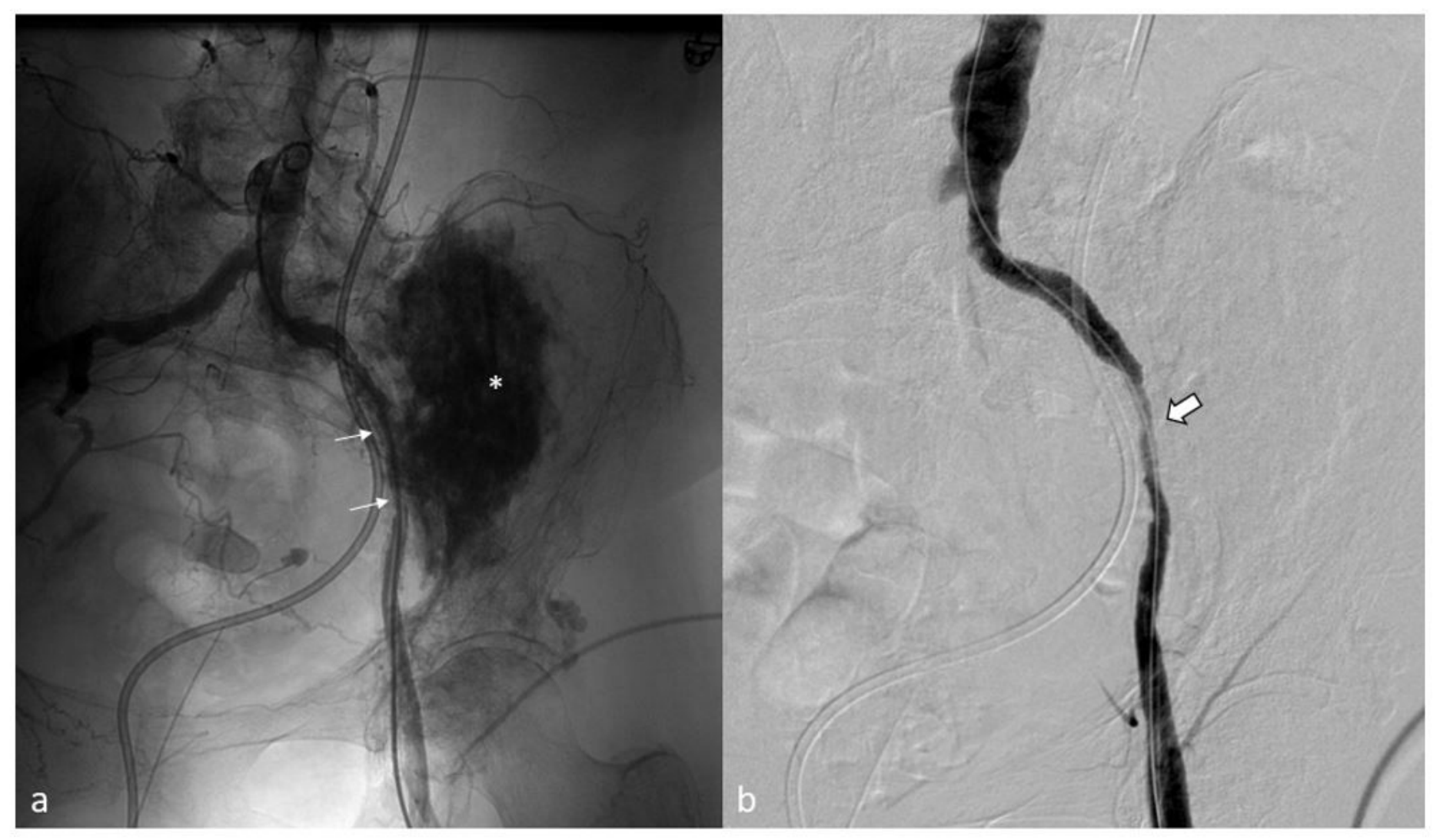

\section{Figure 2}

Case \#3: 81-year-old woman with left sided bleeding from UAF. Angiogram shows the ureteric stent in direct proximity of the left CIA and EIA with closed IIA while there is no direct proof of the fistula on angiography. Massive calcified bone metastasis $\left(^{*}\right)$ compresses the left iliac arteries (small arrows) (a). The final control angiogram shows the crossing of the common iliac artery and the ureteric stent covered by a stent graft. Metastatic compression led to a not fully unfolded stent graft (large arrow) (b).

\section{Supplementary Files}

This is a list of supplementary files associated with this preprint. Click to download.

- Table13.docx 